\title{
Pegcetacoplan in Paroxysmal Nocturnal Haemoglobinuria
}

\author{
Richard J Kelly, Alexandra Pike, Daniel Payne and Morag Griffin \\ Department of Haematology, St James's University Hospital, Leeds, UK
}

DOI: https://doi.org/10.17925/OHR.2021.17.2.84

$\mathrm{P}$ aroxysmal nocturnal haemoglobinuria (PNH) is an acquired stem cell disorder. Patients experience chronic intravascular haemolysis on a background of bone marrow failure, and are at increased risk of thrombosis. Treatment of this condition is focused on complement inhibition to ameliorate the symptoms experienced, which occur due to complement activation. Eculizumab and ravulizumab were approved for the treatment of PNH by the US Food and Drug Administration in 2007 and 2018, respectively. These therapies bind to the complement protein C5, preventing intravascular haemolysis and the symptoms of the disease. However, inhibiting complement at C5 in PNH increases extravascular haemolysis, which leads to anaemia, with some patients requiring transfusions. Pegcetacoplan inhibits complement earlier in the complement pathway at the complement protein C3, preventing intravascular haemolysis but without increasing extravascular haemolysis. This review includes diagnosis and management of PNH, including the new therapy pegcetacoplan.

\section{Keywords}

Paroxysmal nocturnal haemoglobinuria, complement, intravascular haemolysis, extravascular haemolysis, pegcetacoplan

Disclosures: Richard J Kelly has received honoraria from, and served on advisory boards for Alexion Pharmaceuticals and Sobi Pharmaceuticals. Alexandra Pike has received PhD education grant funding from Apellis Pharmaceuticals. Daniel Payne has received honoraria from Alexon Pharmaceuticals. Morag Griffin has received honoraria from, and served on advisory boards for Alexion Pharmaceuticals and Sobi Pharmaceuticals, and Biocryst Pharmaceuticals, and has consulted for Regeneron Pharmaceuticals. Review process: Double-blind peer review.

Compliance with ethics: This study involves a review of the literature and did not involve any studies with human or animal subjects performed by any of the authors.

Data availability: Data sharing is not applicable to this article as no datasets were generated or analysed during the current study.

Authorship: The named authors meet the International Committee of Medical Journal Editors (ICMJE) criteria for authorship of this manuscript, take responsibility for the integrity of the work as a whole and have given final approval for the version to be published.

Access: This article is freely accessible at touchONCOLOGY.com @ Touch Medical Media 2021

Received: 16 August 2021

Accepted: 20 September 2021

Published online: 29 November 2021

Citation: touchREVIEWS in Oncology \& Haematology. 2021;17(2):84-9

Corresponding author: Richard Kelly, Department of Haematology, St James's University Hospital, Beckett Street, Leeds, LS9 7TF, UK. E: richardkelly@nhs.net

Support: No funding was received in the publication of this article.
Paroxysmal nocturnal haemoglobinuria (PNH) is a rare, chronic haematological illness. The condition presents on a background of bone marrow failure, and affected individuals can experience an array of symptoms due to chronic haemolysis, as well as episodes of acute haemolysis and thrombotic events.' Diagnostic delays in PNH are common as not all patients experience classical symptoms, such as haemoglobinuria, at initial presentation. ${ }^{2}$ In 2007, the US Food and Drug Administration (FDA) approved the use of eculizumab for treating PNH. Eculizumab is a humanized monoclonal antibody that stops the formation of terminal complement, thereby preventing intravascular haemolysis. ${ }^{3}$ It effectively reduces morbidity and improves mortality to near-normal expectations. ${ }^{4-7}$

Other complement inhibitors have been developed for treating PNH, with the FDA approving ravulizumab in 2018 and, more recently, in May 2021, pegcetacoplan. This review describes the condition and its treatment, focusing on the rationale for the use of pegcetacoplan.

\section{Disease pathophysiology}

Most cases of PNH are due to a somatic mutation of the phosphatidylinositol glycan A (PIG-A) gene, which is one of over 25 genes required to form the glycophosphatidylinositol (GPI) anchor within the endoplasmic reticulum (ER). ${ }^{8-11}$ The GPI anchor is formed via a stepwise addition of sugar nucleotides and phospholipids within the ER, before the completed protein is transferred to the cell surface. ${ }^{12}$ The GPI anchor holds a wide variety of cell surface proteins that play important roles in signal transduction and the immune response. 13,14 PIG-A mutations disrupt GPI production causing an absence of the GPI anchor and consequently of GPI-linked proteins. CD55 (decay accelerating factor) and CD59 (membrane inhibitor of reactive lysis) are GPI-linked proteins widely expressed on haematopoietic cells and are responsible for regulating complement. PNH red blood cells can be classified as either type II cells, with a reduced expression of CD55 and CD59, or as type III cells, with a complete absence of CD55 and CD59. ${ }^{15}$ Without expressing complement regulatory proteins, PNH red cells are lysed in the circulation (intravascular haemolysis). PNH platelets are not lysed but instead undergo conformational change, whereas PNH leucocytes become activated and release pro-inflammatory cytokines. These effects on PNH cells are implicated in the different symptoms experienced by patients with $\mathrm{PNH}$.

\section{PIG mutations in paroxysmal nocturnal haemoglobinuria}

PIG-A is on the X chromosome and therefore mono-allelically expressed, so only a single mutation in the gene is needed to disrupt GPI formation. All the other genes involved in GPI biosynthesis are autosomal; for other genes to be causative would require both copies of the gene to be mutated. Mutations in other genes, PIG-M, PIG-B and PIG-T, have all been described. ${ }^{16-19}$ PIG-M has been identified in two unrelated consanguineous families. Affected individuals present in childhood with thrombosis and epilepsy. ${ }^{16}$ Four cases with a germline mutation in one PIG-T allele and a somatic mutation in the other PIG-T allele have also been reported..$^{20}$ All four of these PIG-T-mutated cases experienced auto-inflammatory symptoms preceding the clinical 
symptoms of PNH. Langemeijer et al. described a rare familial form of PNH due to a homozygous mutation in PIG-B, with affected family members also experiencing unusual inflammatory symptoms, with inflammatory features commencing just before the diagnosis of $\mathrm{PNH}^{17}$

\section{Paroxysmal nocturnal haemoglobinuria development and classification}

There is a strong association between PNH and bone marrow failure. ${ }^{21-25}$ It is likely that PNH only develops on a background of bone marrow failure, in an environment that allows the PNH clone to expand, as a PIG-A mutation in a haematopoietic stem cell on its own is not enough to cause $\mathrm{PNH}^{26-28} \mathrm{PNH}$ clones have been reported in over $50 \%$ of individuals with aplastic anaemia. ${ }^{28-32}$ However, it is not clear what causes the proportion of these PNH cells to increase in some cases.

In general, the more PNH blood cells are produced, the more likely PNH symptoms will manifest. A classification of PNH was devised in 2005 by Parker et al. on behalf of the International PNH Interest Group with the following categories: classic $\mathrm{PNH}, \mathrm{PNH}$ occurring in the presence of another specified bone marrow disorder, and subclinical PNH. ${ }^{21}$ Patients with classic PNH have evidence of intravascular haemolysis but not of another bone marrow disorder, whilst patients with $\mathrm{PNH}$ in the presence of another bone marrow disorder have intravascular haemolysis, either with a history of, or with another defined bone marrow condition. Subclinical PNH is where a small proportion of PNH cells are present but there is no haemolysis. In clinical practice, there is a significant overlap between the first two categories.

\section{Laboratory diagnosis of paroxysmal nocturnal haemoglobinuria}

Flow cytometric detection is the gold standard method for diagnosing and monitoring $\mathrm{PNH}$. This technique allows evaluation of $\mathrm{PNH}$ neutrophils and erythrocytes down to a level of $0.01 \% .{ }^{33}$ The International Clinical Cytometry Society recommends gating antibodies CD45, CD15 and CD64 for assessing neutrophils and monocytes, combined with fluorescent-labelled proaerolysin (FLAER), CD24, CD14 and CD157 for detecting PNH cells. ${ }^{33}$ FLAER can be used to assess neutrophils and monocytes, as it binds directly to the GPI anchor, which is more specific and sensitive to analyse, rather than to a single GPI-linked protein, of which there are multiple, on the surface of these cells. Assessment of erythrocytes is also recommended using CD235a for gating and CD59 for detecting PNH cells.

It is essential to assess $\mathrm{PNH}$ clones in the white cell lineage to accurately determine the size of the $\mathrm{PNH}$ clone. The proportion of $\mathrm{PNH}$ cells identified in erythrocytes may be lower than in white cells, due to intravascular haemolysis and red cell transfusions required to treat anaemia. Assessing erythrocytes may provide useful clinical information, including the percentage of type II and type III cell populations, and adding C3d to this assay enables rapid flow cytometric assessment of extravascular haemolysis resulting from inhibiting complement at complement protein $\mathrm{C}^{3}{ }^{34}$

\section{Clinical features of paroxysmal nocturnal haemoglobinuria}

Chronic haemolysis is the underlying cause of the complications observed in PNH. Patients experience variable signs and symptoms, including haemoglobinuria, fatigue, dysphagia, abdominal and chest pain, renal impairment, erectile dysfunction and an increased incidence of thrombosis. ${ }^{35}$

\section{Thrombosis}

Prior to complement inhibition, thrombosis was the main cause of mortality in $\mathrm{PNH}$. It may also be the presenting event leading to a diagnosis of $\mathrm{PNH}$. Around $29-40 \%$ of patients will develop thromboses during the course of the disease. ${ }^{1,36-39}$ It is essential to diagnose and promptly treat patients presenting with thrombosis to reduce clot propagation; anticoagulation alone is insufficient in managing a PNH thrombotic event. Thrombotic events commonly manifest as pulmonary emboli and deep vein thromboses, but there is an increase in clot formation in unusual sites, such as the liver (Budd-Chiari syndrome), abdomen and cerebral veins. ${ }^{1,40}$ Fifteen percent of events are arterial. ${ }^{1}$ Thrombotic events occur due to a combination of factors, including intravascular haemolysis, the release of free haemoglobin, platelet and leucocyte activation, and endothelial activation. ${ }^{39}$

\section{Other disease complications}

Renal impairment is present in around two-thirds of patients with PNH. ${ }^{41}$ In most cases, this is mild in nature with stage 1 and 2 chronic kidney disease, but around $20 \%$ of cases are stage 4 or 5 . Renal disease occurs as a result of haemosiderin deposition in the renal tubules, vasoconstriction of blood vessels, and microthrombi.42-44 Nitric oxide uptake also disrupts smooth muscle function, leading to symptoms including dysphagia, abdominal pain, breathlessness and erectile dysfunction. ${ }^{45}$

\section{Treatment of paroxysmal nocturnal haemoglobinuria}

\section{General supportive measures in paroxysmal} nocturnal haemoglobinuria

A diagnosis of PNH can be confusing for patients. Accessing specialist support can be difficult, but both good disease information and patient support groups are helpful. Not all patients with $\mathrm{PNH}$ require pharmacological management. Patients with smaller PNH clones and those without indications for treatment should be managed proactively with folic acid and close monitoring for symptoms, PNH clone changes and the development of other bone marrow failure disorders.

Patients with larger PNH clones who are not indicated for complement inhibition should be considered for prophylactic anticoagulation where safe to do so. In countries where complement inhibition is not available, anticoagulation should also be considered.

\section{Inhibition of complement in $\mathrm{PNH}$}

complement inhibition is generally indicated in patients with symptomatic intravascular haemolysis, PNH-related thrombosis (clinical emergency) or PNH-related longer-term complications, such as renal failure and pulmonary hypertension. Most centres also advocate the use of eculizumab during pregnancy (unlicensed use).

Complement inhibition is not available in many countries worldwide, due to the high cost of the treatment, thus creating a disparity, which, in time, should be addressed by the increasingly competitive nature of the treatment landscape.

\section{Inhibition of complement at C5}

Eculizumab and ravulizumab block terminal complement formation by binding to the complement protein C5 and preventing its cleavage to C5a, an anaphylatoxin, and C5b, which forms part of the membrane attack complex (Figure 1). These therapies can block intravascular haemolysis, thereby reducing disease morbidity and mortality. ${ }^{4-7,46,47}$ 
Figure 1: Complement pathways and inhibitors

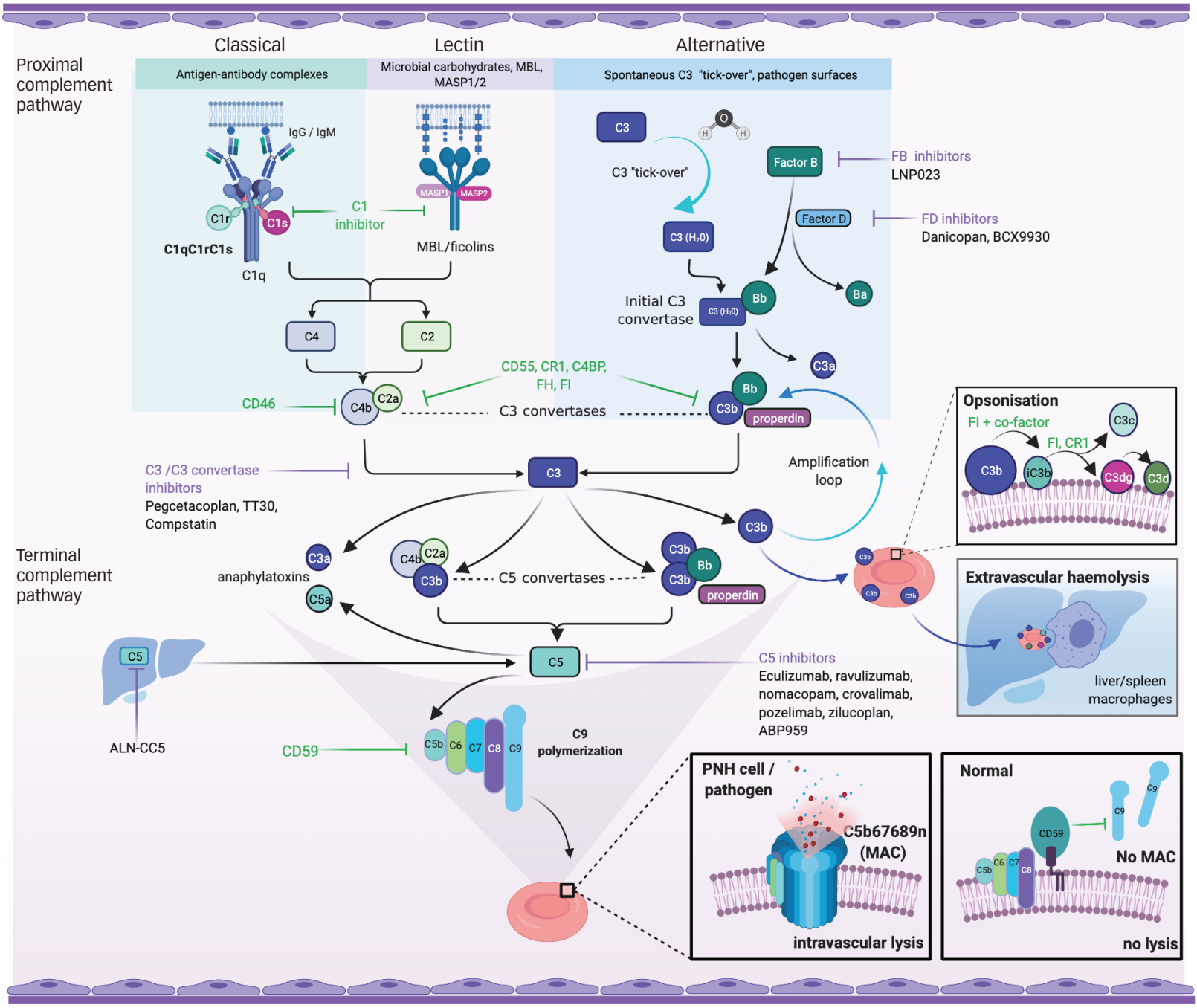

A cascade of proteolytic complement components can be triggered via three principal mechanisms including antigen-antibody interactions (classical pathway), pathogen carbohydrates (lectin pathway) and spontaneous C3 hydrolysis ('tick-over') or pathogen surfaces (alternative pathway). All converge to create the C3 convertase complexes that cleave C3 into catalytically active fragments (C3a and C3b). Further rapid generation of C3b is achieved via the alternative pathway amplification loop. Generation of the C5 convertases results in C5 cleavage into its active components and assembly of the membrane attack complex (MAC) causing lysis of the target cell. The MAC is normally inhibited by CD59 and, consequently, the deficient PNH cells are vulnerable to intravascular haemolysis. Cells not lysed intravascularly may become progressively opsonized with C3b. This is further cleaved by factor I with co-factors to generate ic3b, C3d, C3c and C3d, which serve as ligands for other immune cells and target the cells for extravascular removal in the reticulo-endothelial system. PNH cells are also deficient in CD55, which normally disrupts C3 and C5 convertase activity. Tight control of the system is required to prevent overactivation and host damage. There are many natural regulators (examples in green) and targets for therapeutic agents (examples in purple). $C R 1=$ complement receptor $1 ; F B=$ factor $B ; F D=$ factor $D ; F H=$ factor $H ; F I=$ factor $1 ; M A C=$ membrane attack complex; $M B L=$ mannose binding lectin; $M A S P=$ membrane-associated serine protease; $P N H=$ paroxysmal nocturnal haemoglobinuria. Created with BioRender.com

Eculizumab was approved by the FDA and the European Medicines Agency for the treatment of PNH in 2007 and has been widely used since its approval. Initially, $600 \mathrm{mg}$ is given weekly for the first 4 weeks, and then $900 \mathrm{mg}$ every 2 weeks thereafter..$^{4-6} \mathrm{It}$ has also been used in pregnancy with good maternal and foetal outcomes. ${ }^{48}$

The FDA approved ravulizumab for use in PNH in 2018. Ravulizumab, which has a half-life four times that of eculizumab, is administered according to weight as an initial loading dose, followed by a maintenance dose 2 weeks later, and then every 8 weeks. The loading dose is 2,400 $\mathrm{mg}$ for patients weighing 40-60 kg, 2,700 mg for those weighing 60-100 $\mathrm{kg}$ and $3,000 \mathrm{mg}$ for those weighing over $100 \mathrm{~kg}$. The maintenance dose for these weight ranges is $3,000 \mathrm{mg}, 3,300 \mathrm{mg}$ and $3,600 \mathrm{mg}$, respectively. Phase III trials have demonstrated non-inferiority to eculizumab both in untreated patients and in those who were stable on eculizumab. ${ }^{46,47}$

\section{Complement-driven extravascular haemolysis}

Whilst complement inhibition targeting the complement protein C5 is highly effective in preventing intravascular haemolysis and its consequences, the use of these therapies may lead to an increase in extravascular haemolysis of PNH erythrocytes. Extravascular haemolysis in patients receiving $\mathrm{C} 5$ inhibitors is driven by $\mathrm{C} 3$ fragments opsonizing the $\mathrm{PNH}$ red cells, marking them for destruction within the reticulo-endothelial system (Figure 2). ${ }^{34,49}$ Although anaemia in $\mathrm{PNH}$ can be multifactorial with aplasia also being implicated, extravascular haemolysis is experienced by most patients on $\mathrm{C} 5$ inhibitors, causing anaemia and, in some cases, necessitating blood transfusions. In an analysis of 141 patients maintained on eculizumab for at least 13 months, $72 \%$ were anaemic with $36 \%$ of these requiring at least one transfusion per year. ${ }^{50}$ 
Figure 2: Extravascular haemolysis

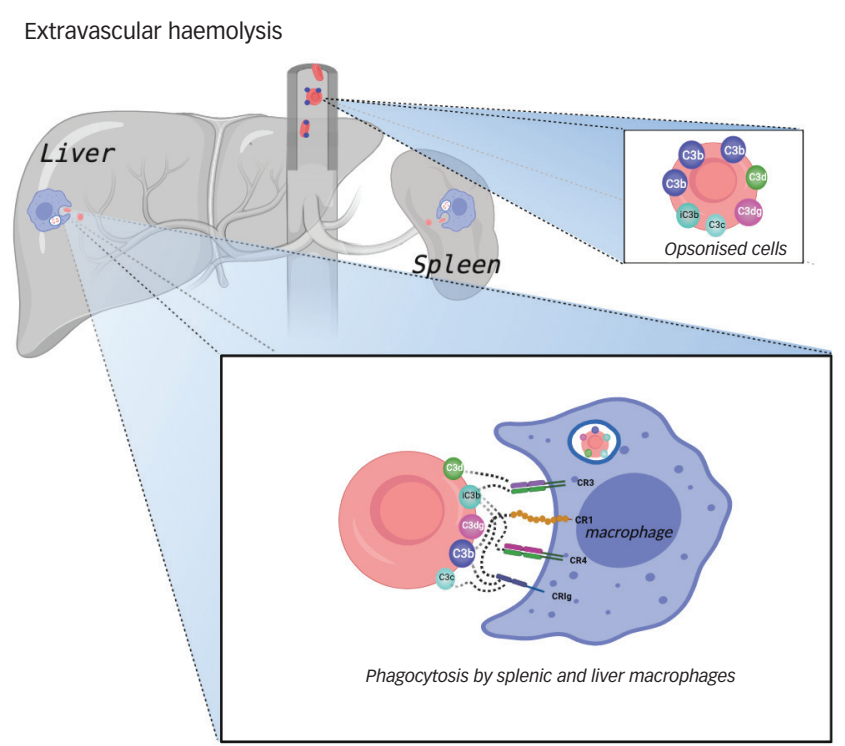

Paroxysmal nocturnal haemoglobinuria red blood cells that are not intravascularly lysed, for example, through C5 inhibitor therapy, may become progressively opsonized with C3b and C3b breakdown fragments (ic3b, C3c, C3dg and C3d). These are ligands for several complement receptors on antigen-presenting cells, B cells and macrophages. Interaction with complement receptors on the reticulo-endothelial system macrophages of the liver and spleen triggers phagocytosis leading to extravascular haemolysis.

CR1, complement receptor 1; CR2, complement receptor 2; CR4, complement receptor 4; CRIg, complement receptor of the immunoglobulin family.

created with BioRender.com

\section{Inhibition of complement at C3: pegcetacoplan}

Pegcetacoplan is a new class of complement inhibitor. It is a pegylated synthetic peptide conjugated to a polyethylene glycol polymer that binds to the complement protein C3 preventing its cleavage to C3a and C3b. Pegcetacoplan is a self-administered, twice-weekly subcutaneous injection $(1,080 \mathrm{mg})$, and prevents both intravascular and extravascular haemolysis by preventing C3 opsonization of PNH red blood cells (Figure 3).

De Castro et al. assessed the safety, efficacy and pharmacokinetics of pegcetacoplan in a phase I trial of patients who continued to experience anaemia despite eculizumab therapy..$^{51}$ Nine patients were enrolled, with four completing the study and continuing on pegcetacoplan in an extension study. Pegcetacoplan was well tolerated, with increases in haemoglobin and decreases in bilirubin and reticulocyte levels observed.

The key 16-week trial data are from the phase III, open-label clinical trial (Pegasus trial; ClinicalTrials.gov identifier NCT03500549), which evaluated the efficacy and safety of pegcetacoplan compared with eculizumab. ${ }^{52}$ Adult patients who were diagnosed with PNH by flow cytometry and who had a haemoglobin level $<10.5 \mathrm{~g} / \mathrm{dL}$ whilst receiving stable doses of eculizumab for at least 3 months were eligible for the study. The trial was divided into three distinct parts, with a 4-week 'run-in' period where patients received their previous eculizumab therapy as well as twice-weekly pegcetacoplan (1,080 mg per injection). After the runin period, patients were randomized 1:1 to receive either eculizumab or pegcetacoplan for 16 weeks, followed by a 32-week open-label extension where all were treated with pegcetacoplan. During the openlabel extension, the patients from the eculizumab group were treated with eculizumab and pegcetacoplan for the initial 4 weeks, followed by 28 weeks of pegcetacoplan monotherapy. The 1:1 randomization was stratified for platelet count higher or lower than $100 \times 10^{\circ} / \mathrm{L}$, and greater or fewer than four transfusions in the preceding 12 months prior to enrolment in the trial.

The primary endpoint of the trial was the change in haemoglobin level from baseline to week 16. Secondary endpoints measured at week 16 included the proportion of patients who were transfusion independent, the change in reticulocyte and lactate dehydrogenase (LDH) levels and changes in the functional assessment of chronic illness therapy-fatigue (FACIT-F) score. Eighty patients (39 female, 41 male) were treated in the study, with 41 in the pegcetacoplan arm and 39 in the eculizumab arm. Three of those in the pegcetacoplan arm discontinued the study due to experiencing breakthrough haemolysis (BTH). The characteristics of the patients in the two arms were largely similar, including patient age, body mass index, prior history of aplastic anaemia, haemoglobin, platelet count, LDH, FACIT-F score and median duration on eculizumab. Twenty-four (30\%) patients were receiving higher than standard doses of eculizumab at the start of the trial.

The primary endpoint was achieved, with a mean increase in haemoglobin in the pegcetacoplan arm of $2.37 \mathrm{~g} / \mathrm{dL}$ compared with a reduction in the eculizumab arm of $1.47 \mathrm{~g} / \mathrm{dL}$ at 16 weeks. Regarding the main secondary endpoints, significantly more patients were transfusion independent in the pegcetacoplan arm (35/41, 85\%) when compared with the eculizumab arm $(6 / 39,15 \%)$ over the 16 -week period. A clinically significant improvement in FACIT-F scores at week 16 was also observed in those receiving pegcetacoplan, with a 9.2 point increase compared with a 2.7 point decrease in those receiving eculizumab. Non-inferiority of pegcetacoplan was seen in absolute reticulocyte count levels, which decreased on pegcetacoplan and increased slightly on eculizumab $\left(-136 \pm 7 \times 10^{\circ} / \mathrm{L}\right.$ versus $28 \pm 12 \times 10^{\%} / \mathrm{L}$, respectively). Non-inferiority was not seen in the mean change in LDH from baseline $(15 \pm 43 \mathrm{U} / \mathrm{L}$ with pegcetacoplan compared with $10 \pm 71 \mathrm{U} / \mathrm{L}$ with eculizumab). More patients' blood values became normal on pegcetacoplan compared with eculizumab; haemoglobin (34\% versus $0 \%$ ), LDH (71\% versus 15\%), reticulocytes (78\% versus $3 \%$ ) and bilirubin (63\% versus $8 \%$ ).

Adverse events occurred in a similar proportion of patients on pegcetacoplan as on eculizumab. Roughly equal levels of infections were seen, occurring in $29 \%$ on pegcetacoplan and $26 \%$ on eculizumab. No meningococcal or pyogenic infections were observed during the trial; however, follow-up was very short. Episodes of BTH occurred in four patients (10\%) on pegcetacoplan and in nine (23\%) on eculizumab. The other common adverse events on pegcetacoplan were injection site reactions (37\%) and diarrhoea (22\%). Pegcetacoplan has also been approved by the FDA for treatment-naive patients with $\mathrm{PNH}$ who require complement inhibition.

\section{Managing breakthrough haemolysis on proximal complement inhibitors}

Episodes of BTH in patients treated with proximal complement inhibitors are potentially difficult to manage. Due to improvements in haemoglobin levels and consequently the proportion of PNH red cells, a stimulating event, such as infection, can result in brisk haemolysis and a rapid drop in haemoglobin. Whilst the degree of BTH can be marked, an additional dose of a C5 inhibitor can stop the haemolytic episode. Within the Pegasus trial, four patients on pegcetacoplan experienced BTH in the 16 -week period. ${ }^{52}$ Whilst nine patients on eculizumab experienced breakthrough in the first 16 weeks, these events could be managed with additional C5 inhibition (outside of the clinical trial setting).

A strategy for managing BTH events on all proximal complement inhibitors, not just pegcetacoplan, is essential. This strategy should 
Figure 3: Flow cytometry dot plots demonstrating C3d deposition on PNH erythrocytes

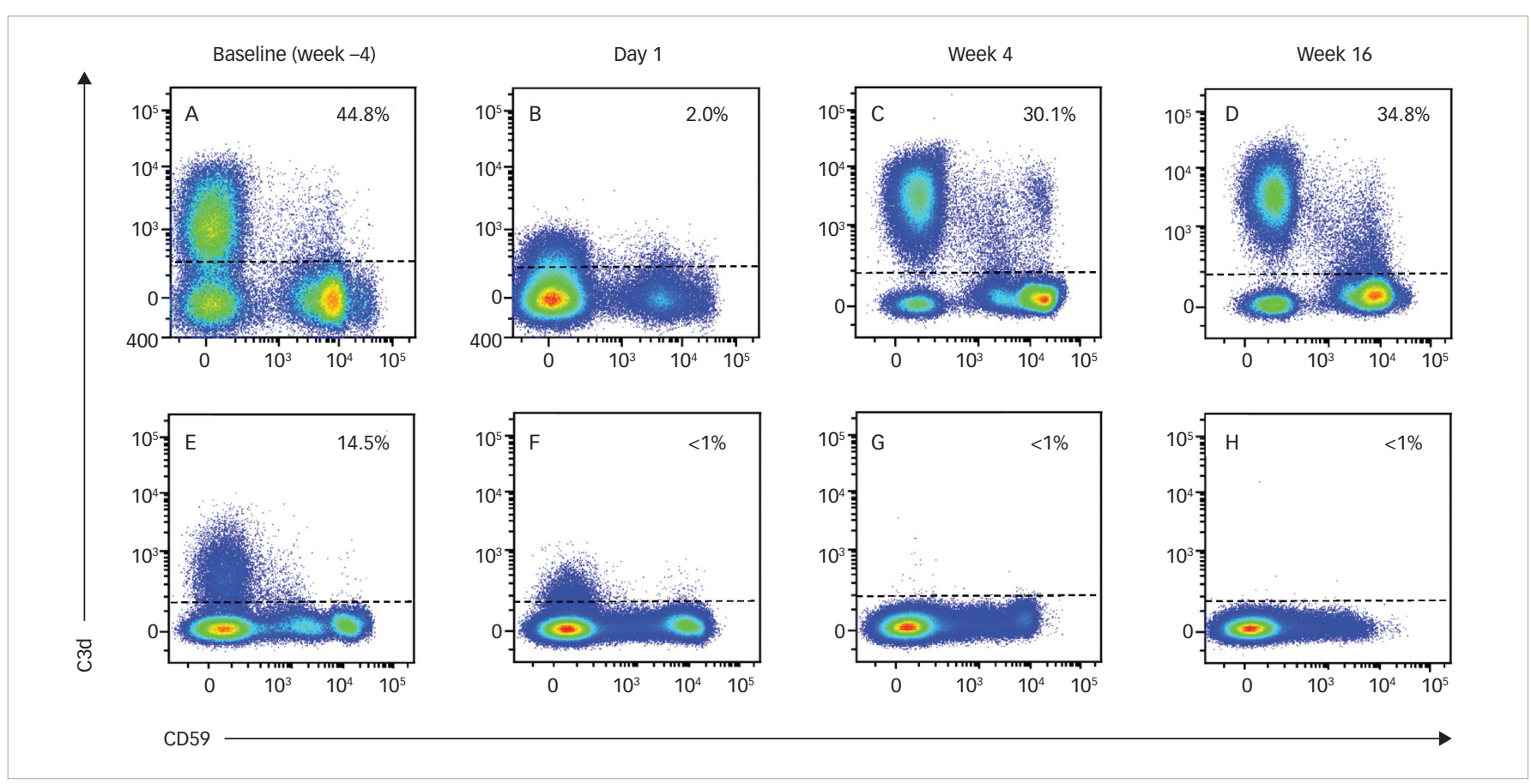

A-D: show a patient treated with eculizumab; E-H: show a patient treated with pegcetacoplan. C3d was predominantly observed on CD59-negative (type III) erythrocytes. Percentage of C3d deposition on total erythrocytes is shown in the top right of each dot plot. Significantly reduced C3d deposition was demonstrated in patients on pegcetacoplan throughout the duration of therapy.

Peripheral blood was diluted 1:100, and $50 \mu \mathrm{L}$ was incubated for 15 minutes with CD235a-FITC, CD59-PE and C3d-Biotin. Cells were then washed with filtered saline, Streptavadin-APC was added, and incubated for a further 15 minutes. Two further washing cycles were performed and cells were vigorously mixed (by racking). The final volume was resuspended to $0.5 \mathrm{~mL}$ and a minimum of 500,000 CD235a positive erythrocyte events were acquired on FACSLyric flow cytometer (Becton Dickinson). Data were analysed with Kaluza analysis software v2.1 (Beckman Coulter). All plots shown in the figure were gated on CD235a-positive erythrocytes. Each column represents different treatment time points.

include good patient education, particularly for patients who may have experienced BTH on C5 inhibition, as symptoms can be different and haemolysis can be brisk. How to manage these events pharmacologically can be debated; for patients with suboptimal LDH control $(>2 \times$ upper limit of normal), the dose of pegcetacoplan can be increased to 1,080 mg every 3 days; however, this may not be sufficient, particularly during an acute haemolytic breakthrough, and an alternative treatment might be required, such as a C5 inhibitor.

\section{Managing infection risk on complement inhibition}

Individuals with a hereditary deficiency of C5 are unable to initiate an immune response to meningococcal infection, and complement inhibition provides a similar risk. Educating patients and their families is essential, as is vaccinating against meningococcal serotypes ACWY and B. Some centres advocate the monitoring of antibody titres to review and optimize the immune response, particularly to the ACWY vaccine (it is not possible to assess titres for serotype B due to C5 inhibition interfering with the assay), and providing meningitis B boosters every 5 years. ${ }^{53}$ Proximal complement inhibition raises the possibility of increased risk of infection with other encapsulated organisms, and thus it is advised that patients are vaccinated against Haemophilus and pneumococci alongside meningococcal vaccinations. Some centres also promote the use of prophylactic antibiotics and a 'back-up' emergency provision with ciprofloxacin, as well as prompt medical reviews if pyrexial.

\section{Other proximal complement inhibitors}

Whilst not the main discussion for this article, other proximal complement inhibitors are in clinical trials or early stages of development, including targeting factor $\mathrm{B}$ and factor D. ${ }^{54,55}$

\section{How to choose the treatment option most suitable for your patient}

Where possible, all patients with PNH should be offered entry into a clinical trial. For those commencing approved therapies, which treatment option to choose for your patient may become more complex. Current licensed treatments in the USA include eculizumab, ravulizumab and pegcetacoplan.

Data from the Pegasus trial support the use of pegcetacoplan in those with significant extravascular haemolysis on a C5 inhibitor. However, for those patients well controlled on C5 inhibitors, whilst the option to change to pegcetacoplan should be considered, there are merits in remaining on a C5 inhibitor. Data on pegcetacoplan in $\mathrm{PNH}$ are still in their infancy in terms of longer-term complications and thrombotic events. In individuals with a complicated thrombotic history, or those presenting with thrombosis as their diagnostic event for $\mathrm{PNH}$, it is likely preferable to use C5 inhibition, at least initially.

Thrombocytopaenia, when severe, can mean pegcetacoplan is not suitable, as pegcetacoplan is administered subcutaneously. In women with $\mathrm{PNH}$ considering pregnancy, eculizumab would be the preferred treatment until more data are available in this setting. ${ }^{48}$

\section{Conclusion}

The treatment of PNH has been revolutionized by complement inhibition. Prior to eculizumab, patients were managed with supportive care and, in some cases, allogeneic bone marrow transplantation. ${ }^{1}$ By preventing terminal complement formation, eculizumab and ravulizumab reduce the complications of chronic intravascular haemolysis, thereby improving patient outcomes and quality of life. ${ }^{4-6,46,47}$ Despite this 
improvement, over $70 \%$ of patients on eculizumab remain anaemic, with some requiring transfusions due to extravascular haemolysis. ${ }^{50}$

Pegcetacoplan, by blocking complement earlier in the complement pathway, prevents intravascular haemolysis without causing an increase in extravascular haemolysis. ${ }^{52}$ This therapy, like C5 inhibitors, prevents the unwanted effects of intravascular haemolysis, but when compared with eculizumab, improves haemoglobin levels and, consequently, FACIT-F scores.

Whilst pegcetacoplan is a promising therapy for treating $\mathrm{PNH}$, there is still far more clinical experience in C5 complement inhibitors. How to manage BTH, and how to manage patients presenting with a thrombosis or with complicated thromboses remain issues about which we will continue to learn and gather answers. Concerns regarding infection risk, in particular the increased risk of meningococcal infection, should be proactively managed.

The treatment landscape for $\mathrm{PNH}$ has changed significantly with newer therapies becoming available. The options for patients in a few years' time may allow for an individualized approach to treatment. With oral, subcutaneous and long-acting intravenous options possible, we hope that pricing structure will be improved, widening access to patients worldwide who have indications for complement inhibition.

Pegcetacoplan is the first proximal complement inhibitor to be approved for use in PNH by the FDA. In patients who have ongoing anaemia due to extravascular haemolysis on C5 inhibition, pegcetacoplan may be a better therapy.
1. Hillmen P, Lewis SM, Bessler M, et al. Natural history of paroxysmal nocturnal hemoglobinuria. N Eng/ J Med. 1995;333:1253-8

2. Dacie JV, Lewis SM. Paroxysmal nocturnal haemoglobinuria: clinical manifestations, haematology, and nature of the disease Ser Haematol. 1972:5:3-23.

3. Thomas TC, Rollins SA, Rother RP, et al. Inhibition of complement activity by humanized anti-C5 antibody and single-chain Fv. Mol Immunol. 1996;33:1389-1401.

4. Hillmen P, Hall C, Marsh JC, et al. Effect of eculizumab on hemolysis and transfusion requirements in patients with paroxysmal nocturnal hemoglobinuria. N Eng/ J Med. 2004;350:552-9.

5. Hillmen P, Young N, Schubert J, et al. The complement inhibitor eculizumab in paroxysmal nocturnal hemoglobinuria. N Eng/ 」 Med. 2006:355:1233-43.

6. Brodsky RA, Young NS, Antonioli E, et al. Multicenter phase 3 study of the complement inhibitor eculizumab for the treatment of patients with paroxysmal nocturnal for the treatment of patients with paroxys

7. Kelly RJ, Hill A, Arnold LM, et al. Long-term treatment with eculizumab in paroxysmal nocturnal hemoglobinuria: sustained efficacy and improved survival. Blood. 2011;117:6786-92.

Takeda J, Miyata T, Kawagoe K, et al. Deficiency of the GP anchor caused by a somatic mutation of the PIG-A gene in paroxysmal nocturnal hemoglobinuria. Cell. 1993;73:703-11.

9. Miyata T, Takeda J, lida Y, et al. The cloning of PIG-A, a component in the early step of GPI-anchor biosynthesis. Science. 1993;259:1318-20.

10. Miyata T, Yamada N, lida Y, et al. Abnormalities of PIG-A transcripts in granulocytes from patients with paroxysma nocturnal hemoglobinuria. N Eng/ I Med. 1994:330:249-55.

11. Bessler M Mason PJ, Hillmen P et al. Paroxysmal nocturn haemoglobinuria (PNH) is caused by somatic mutations in the PIG-A gene. EMBO J. 1994:13:110-7.

12. Vidugiriene J, Menon AK. Early lipid intermediates in glycosylphosphatidylinositol anchor assembly are synthesized in the ER and located in the cytoplasmic leaflet of the ER membrane ER and located in the cytoplasmic leafl
bilayer. $J$ Cell Biol. 1993:121:987-96.

13. Mahoney JF, Urakaze M, Hall S, et al. Defective glycosylphosphatidylinositol anchor synthesis in paroxysmal nocturnal hemoglobinuria granulocytes. Blood. 1992;79:1400-3

14. Paulick MG, Bertozzi CR. The glycosylphosphatidylinositol anchor: a complex membrane-anchoring structure for proteins. Biochemistry. 2008;47:6991-7000.

15. Rosse WF. Variations in the red cells in paroxysmal nocturnal haemoglobinuria. Br J Haematol. 1973;24:327-42.

16. Almeida AM, Murakami Y, Layton DM, et al. Hypomorphic promoter mutation in PIGM causes inherited glycosylphosphatidylinositol deficiency. Nat Med. 2006;12:84650 .

17. Langemeijer S, Schaap C, Preijers F, et al. Paroxysmal nocturnal hemoglobinuria caused by CN-LOH of constitutional PIGB mutation and 70-kbp microdeletion on 15q. Blood Adv. 2020;4:5755-61

18. Krawitz PM, Höchsmann B, Murakami Y, et al. A case of paroxysmal nocturnal hemoglobinuria caused by a germline mutation and a somatic mutation in PIGT. Blood. 2013;122:1312-15

19. Kawamoto M, Murakami Y, Kinoshita T, et al. Recurrent aseptic meningitis with PIGT mutations: a novel pathogenesis of recurrent meningitis successfully treated by eculizumab. $B M$ Case Rep. 2018;2018:bcr-2018-225910.
20. Höchsmann B, Murakami Y, Osato M, et al. Complement and inflammasome overactivation mediates paroxysmal nocturnal hemoglobinuria with autoinflammation. J Clin Invest. 2019:129:5123-36.

21. Parker $\mathrm{C}$, Omine $\mathrm{M}$, Richards $\mathrm{S}$, et al. Diagnosis and management of paroxysmal nocturnal hemoglobinuria. Blood 2005;106:3699-709.

22. Dameshek W. Riddle: what do aplastic anemia, paroxysmal nocturnal hemoglobinuria (PNH) and 'hypoplastic' leukemia have in common? Blood 1967:30:251-4.

23. Young NS, Maciejewski JP, Sloand E, et al. The relationship of aplastic anemia and PNH. Int J Hematol. 2002;76:168-72.

24. Griscelli-Bennaceur A, Gluckman E, Scrobohaci ML, et al. Aplastic anemia and paroxysmal nocturnal hemoglobinuria: search for a pathogenetic link. Blood. 1995;85:1354-63.

25. Schrezenmeier $\mathrm{H}$, Hertenstein $\mathrm{B}$, Wagner $\mathrm{B}$, et al. $\mathrm{A}$ pathogenetic link between aplastic anemia and paroxysmal nocturnal hemoglobinuria is suggested by a high frequency of aplastic anaemia patients with a deficiency of phosphatidylinositol glycan anchored proteins. Exp Hematol. 1995:23:81-7.

26. Araten DJ, Nafa K, Pakdeesuwan K, et al. Clonal populations of hematopoietic cells with paroxysmal nocturnal hemoglobinuria genotype and phenotype are present in normal individuals Proc Natl Acad Sci USA. 1999;96:5209-14.

27. Araten DJ, Bessler M, McKenzie S, et al. Dynamics of hematopoiesis in paroxysmal nocturnal hemoglobinuria (PNH) no evidence for intrinsic growth advantage of $\mathrm{PNH}$ clones. Leukemia. 2002;16:2243-8.

28. Mukhina GL, Buckley JT, Barber JP, et al. Multilineage glycosylphosphatidylinositol anchor-deficient haematopoiesis in untreated aplastic anaemia. Br J Haematol. 2001;115:476-82.

29. Schubert J, Vogt $\mathrm{HG}$, Zielinska-Skowronek M, et al. Development of the glycosylphosphatitylinositol-anchoring defect characteristic for paroxysmal nocturnal hemoglobinuria in patients with aplastic anemia. Blood. 1994;83:2323-8.

30. Tichelli A, Gratwohl A, Nissen C, et al. Late clonal complications in severe aplastic anemia. Leuk Lymphoma. 1994;12:167-75.

31. Dunn DE, Tanawattanacharoen P, Boccuni P, et al. Paroxysmal nocturnal hemoglobinuria cells in patients with bone marrow failure syndromes. Ann Inter Med. 1999;131:401-8.

32. Wang $\mathrm{H}$, Chuhjo $\mathrm{T}$, Yasue $\mathrm{S}$, et al. Clinical significance of a minor population of paroxysmal nocturnal hemoglobinuria-type cells in bone marrow failure syndrome. Blood. 2002;100:3897-902.

33. Illingworth AJ, Marinov I, Sutherland DR. Sensitive and accurate identification of PNH clones based on ICCS/ESCCA PNH Consensus Guidelines - a summary. Int I Lab Hematol. 2019:41 Suppl. 1:73-81.

34. Risitano AM, Notaro $R$, Marando L, et al. Complement fraction 3 binding on erythrocytes as additional mechanism of disease in paroxysmal nocturnal hemoglobinuria patients treated by in paroxysmal nocturnal hemoglobinuria pa

35. Schrezenmeier $H$. Muus P, Socié $G$, et al. Baseline characteristics and disease burden in patients in the International Paroxysmal Nocturnal Hemoglobinuria Registry. Haematologica. 2014;99:922-9.

36. Nishimura JI, Kanakura Y, Ware RE, et al. Clinical course and flow cytometric analysis of paroxysmal nocturnal hemoglobinuria in the United States and Japan. Medicine (Baltimore). 2004;83:193-207.

37. de Latour RP, Mary JY, Salanoubat C, et al. Paroxysmal nocturna hemoglobinuria: natural history of disease subcategories. Blood. 2008;112:3099-106.
38. Araten DJ, Thaler HT, Luzzatto L. High incidence of thrombosis in African-American and Latin-American patients with paroxysma nocturnal haemoglobinuria. Thromb Haemost. 2005;93:88-91.

39. Hill A, Kelly RJ, Hillmen P. Thrombosis in paroxysmal nocturna hemoglobinuria. Blood. 2013;121:4985-96.

40. Ziakas PD, Poulou LS, Rokas GI, et al. Thrombosis in paroxysmal nocturnal hemoglobinuria: sites, risks, outcome. An overview. Thromb Haemost. 2007;5:642-5.

41. Hillmen P, Elebute M, Kelly R, et al. Long-term effect of the complement inhibitor eculizumab on kidney function in patients with paroxysmal nocturnal hemoglobinuria. Am J Hematol. 2010;85:553-9.

42. Tanaka YO, Anno I, Itai Y, et al. Paroxysmal nocturnal hemoglobinuria: MR findings. I Comput Assist Tomogr. 1993; 17:749-53

43. Rimola J, Martín J, Puig J, et al. The kidney in paroxysma nocturnal haemoglobinuria: MRI findings. Br J Radiol. 2004:77:953-6.

44. Mulopulos GP, Turner DA, Schwartz MM, et al. MRI of the kidneys in paroxysmal nocturnal hemoglobinuria. $A m\rfloor$ Roentgenol 1986:146:51-2.

45. Hill $A$, Rother RP, Wang $X$, et al. Effect of eculizumab on haemolysis-associated nitric oxide depletion, dyspnoea, and measures of pulmonary hypertension in patients with consumption in patients with paroxysmal nocturna haemoglobinuria. Br J Haematol. 2010;149:414-25.

46. Lee JW, Sicre de Fontbrune F, Wong Lee Lee L, et al. Ravulizumab (ALXN1210) vs eculizumab in adult patients with PNH naive to complement inhibitors: the 301 study. Blood. 2019;133:530-9

47. Kulasekararaj $A G$, Hill A, Rottinghaus ST, et al. Ravulizumab (ALXN1210) vs eculizumab in C5-inhibitor-experienced adult patients with PNH: the 302 study. Blood. 2019;133:540-9.

48. Kelly RJ, Höchsmann B, Szer I, et al. Eculizumab in pregnant patients with paroxysmal nocturnal hemoglobinuria. N Eng/ J Med. 2015;373:1032-9.

49. Hill A Rother RP Arnold L, et al. Eculizumab prevents intravascular hemolysis in patients with paroxysmal nocturnal hemoglobinuria and unmasks low-level extravascula hemolysis occurring through C3 opsonization. Haematologica. 2010;95:567-73

50. MCKinley CE, Richards SJ, Munir T, et al. Extravascula hemolysis due to $\mathrm{C} 3$-loading in patients with PNH treated with eculizumab: defining the clinical syndrome. Blood. 2017;130 Suppl. 1:3471.

51. de Castro C, Grossi F, Weitz IC, et al. C3 inhibition with pegcetacoplan in subjects with paroxysmal nocturnal hemoglobinuria treated with eculizumab. Am I Hematol. 2020;95:1334-43.

52. Hillmen P, Szer J, Weitz I, et al. Pegcetacoplan versus eculizumab in paroxysmal nocturnal hemoglobinuria. N Eng/ Med. 2021:384:1028-37.

53. Arnold L, Borrow R, Riley K, et al. Management of meningococcal disease risk in patients with paroxysmal nocturnal hemoglobinuria (PNH) on complement inhibitors: 18 years' experience from the UK national PNH service in Leeds. Blood. 2020;136 Suppl. 1:5-6.

54. Wiles JA, Galvan MD, Podos SD, et al. Discovery and development of the oral complement factor $\mathrm{D}$ inhibitor danicopan (ACH-4471). Curr Med Chem. 2020;27:4165-80.

55. Schubart A, Anderson K, Mainolfi N, et al. Small-molecule factor B inhibitor for the treatment of complement-mediated diseases. PNAS. 2019;116:7926-31. 